\title{
EL LIBRO DE TEXTO UNIVERSITARIO. UN ENFOQUE METODOLÓGICO
}

\author{
THE COLLEGE TEXTBOOK. \\ A METHODOLOGICAL APPROACH
}

\author{
Enaidy Reynosa Navarro* \\ Universidad César Vallejo, Perú
}

\section{RESUMEN}

En el presente trabajo, el lector encontrará pautas metodológicas para la realización de un libro de texto universitario; se sistematizan procedimientos para la conformación del equipo de trabajo encargado de la elaboración de los libros de texto; se explica cómo delimitar las temáticas que pueden ser abordadas; se exponen los procedimientos de gestión de la información, así como la detección de la literatura apropiada. Además, se enfatiza en las formas de indagación teórica, interpretación de teorías, conclusiones teóricas y las funciones didácticas del libro de texto. Por último, se exponen algunas pautas editoriales que van, desde la estructura del libro de texto, notas, referencias bibliográficas, tratamiento de imágenes o tablas, semblanza de los autores, etapa de corrección; hasta la revisión final y puesta en valor de la obra.

Palabras clave: libro de texto universitario, gestión del conocimiento, edición de textos.

\begin{abstract}
In this article, the reader will find methodological guidelines on how to write a college textbook. It offers procedures for setting up a work team responsible for the preparation of textbooks; explains how to define the topics to be addressed, as well as procedures for information management, and detection of appropriate literature. Additionally, it emphasizes theoretical research types, interpretation of theories, theoretical conclusions and the pedagogical function of the college textbook. Finally, it provides some editorial guidelines, such as textbook structure, notes, bibliographical references, images and charts, authors' biographies, correction stage, and final review up to distribution.
\end{abstract}

Keywords: college textbook, knowledge management, text editing. 


\section{INTRODUCCIÓN}

De acuerdo con Acero (2008), los textos universitarios desempeñan un papel fundamental en la educación, puesto que estructuran los procesos de enseñanza $y$ aprendizaje; tienen una decisiva influencia, tanto sobre profesores como en estudiantes; siendo no solo fuente de contenido curricular, sino también una exposición organizada, en una estructura de un cuerpo de conocimientos, junto a un implícito estilo didáctico.

Sin embargo, la realidad actual dista de lo planteado anteriormente y materiales, compendios, separatas, módulos, guías de estudio, etc., que en muchos casos se elaboran de manera descontrolada y sin esa calidad académica corroborada, difieren de los intereses y necesidades del estudiantado. De manera que, "los análisis e investigaciones muestran libros plagados de errores conceptuales y estereotipos ideológicos, además de no haber sido, en la mayor parte de los casos, experimentados y evaluados previamente a su presentación" (Martínez, 2006, p. 63).

En su tesis doctoral, Fernández (2006) explica que la mayoría de los libros de texto padecen de impotencia para promover la motivación de forma intrínseca, debido a su formato estandarizado y su comunicación unidireccional. Agrega que no se respeta el principio de atención a la diversidad, y que las funciones principales son instructivas (para suscitar la recordación y aplicación de lo que se ha leído) y evaluativas.

Martínez (2008), en el artículo "Los libros de texto como práctica discursiva" advierte que estos deben estar despojados del carácter estático, acabado y cerrado, donde no hay dialéctica ni reconstrucción crítica. Explica que se necesitan libros de texto sin la segmentación tradicional académica, que se genera cuando el saber se organiza desde la academia y se plantea tal como es elaborado en la propia academia. Por el contrario, se necesitan libros que prioricen la complejidad, la búsqueda de relaciones, la interacción disciplinar, la interconexión de saberes científicos, el conocimiento compartido, la mirada globalizadora, así como otros saberes que también sirvan para construir y desarrollar la cultura aunque no estén en el interior de las disciplinas tradicionales de la academia.

Las falencias, descritas anteriormente, hacen que el pedagogo recurra a otras fuentes para no incurrir en la desacertada orientación del educando; pero el problema persiste y se evidencia en la escasa producción de libros de texto congruentes con las necesidades del alumno, docente y contexto sociocultural. Por tanto, se impone la necesidad de pensar libros de texto universitarios relevantes e integrales, más parecidos al contexto sociocultural, donde se junten en un todo función instructiva, desarrollo académico, afectivo y social entre alumnos, docentes y sociedad.

Todo este conjunto de problemas mencionados, que como ya se citó son recurrentes en el escenario educativo actual, fueron la motivación principal de este estudio.

\section{Fundamentos del libro de texto}

Antes de ofrecer alguna fundamentación al respecto, se aclara que no es objetivo principal del presente artículo teorizar demasiado en ese sentido, sino acercar al lector beneficiario ideas más esclarecedoras que rebuscadas.

Un estudio publicado por la Facultad de Filosofía y Letras, de la Universidad Nacional Autónoma de México, enfoca el libro de texto desde tres perspectivas fundamentales. La primera, alude al libro de texto como 
objetivación cultural del currículo en todas sus dimensiones; la segunda, como constructor de nuevas concepciones y prácticas sobre su uso en la educación y, finalmente, como aquel que objetiva las relaciones entre discursos y representaciones sociales (Escolano, 2000, citado por Celis, 2001, p. 3). Mientras que para Fernández (2006), los libros de texto "son los únicos libros obligatorios para la sociedad". Son el "pretexto visible y concreto que permite indagar en las dificultades que encuentran los docentes para transformar su enseñanza en una acción social liberadora para ellos y sus educandos" (p. 183).

Una nueva visión de los libros de texto se está desarrollando, principalmente, en Estados Unidos de América, donde los open textbooks o libros de texto abiertos comienzan a tener mayor protagonismo en el escenario educativo de esa nación. Se trata de un libro gratuito, accesible desde internet y elaborado de acuerdo con las necesidades educativas que demanda un proceso de enseñanza-aprendizaje contextualizado. Según el Grupo de Interés Público de Investigación (PIRGs), los open textbooks son textos universitarios gratuitos en línea, de alta calidad y circulación. Bajo esta modalidad, los libros pueden ser legalmente adaptados por los docentes, agregar nuevos materiales, cambiar terminologías o eliminar capítulos innecesarios de acuerdo a sus intereses (Student PIRGs, 2012).

Un libro de texto es "un mediador entre maestro-alumno" (Celis, 2001, p. 1). Un "material específico de enseñanza utilizado por un profesor en el proceso de organizar el trabajo de enseñanza aprendizaje con un grupo o colectivo de estudiantes" (Martínez, 2008, p. 62). Es ese material de consulta multilateral, dialéctico, didáctico, práctico y flexible; basado en cogniciones actualizadas, a tono con la tecnología y con una profunda visión de las problemáticas del contexto sociocultural y del futuro, que favorece la formación académica, profesional y axiológica entre educandos, docentes y sociedad.

\section{PROPUESTA METODOLÓGICA}

La propuesta, que se presenta a continuación, está conformada por una serie de pasos, cuyos objetivos son organizar todo el proceso editorial del libro de texto universitario y proponer los procedimientos que demanda este ejercicio académico.

\section{Conformación del equipo de trabajo}

Conformar el equipo de trabajo es uno de los momentos más importantes, pues aquí puede decidirse o definirse el éxito de la obra que se pretende crear. Para ello, es necesario asegurar que los docentes seleccionados sean aquellos que, desde una visión integral, reúnan las condiciones idóneas, o sea, que tengan un dominio pleno de los aspectos teóricos que fundamentarán la obra, así como de los procedimientos metodológicos que demanda esta tarea. Se necesitan docentes conscientes, que concilien constantemente en pos de "una comunicación y exploración participativas, que dan como resultado la innovación" (Edmondson \& Nembhard, 2010, p. 67). En fin, "los retos del trabajo en equipo pueden ser enormes; pero si se sortean con habilidad, esos mismos retos pueden ayudar a desarrollar la capacidad de adaptación..." (p. 65), creatividad e innovación.

A partir de lo expuesto anteriormente, se proponen procedimientos para la conformación del equipo de trabajo:

a) Hacer una preselección curricular de aquellos profesionales con potencialidades para participar en el proceso de confección de la obra. Dicha 
preselección pude ser el resultado de evaluaciones anteriores hechas por la institución donde se desempeñan y no se recomienda convocar a quienes no cuenten con la experiencia comprobada.

b) Nombrar un equipo multidisciplinario para evaluar los currículos (documentados), preseleccionados. Este equipo debe estar conformado por profesionales con demostrada solvencia académica y, en ningún caso, el seleccionador puede ser autor.

c) El equipo multidisciplinario puede seleccionar directamente a determinados docentes (es recomendable no más de dos). Esto sucede cuando los seleccionados directos gozan de una trayectoria académica probada y documentada.

d) Luego de un proceso exhaustivo de evaluación, tanto del currículo como de las competencias del aspirante, se deben escoger los docentes cuyas trayectorias se ajustan a los requerimientos.

e) Informar por escrito a los docentes seleccionados.

f) Presentar el equipo de trabajo aprobado ante la dirección inmediata superior.

g) Realizar el plan de trabajo donde se definen roles, así como el monitoreo del cumplimiento de los mismos a corto, mediano y largo plazo.

\section{Temáticas de la obra}

Para planificar las temáticas que abordará el libro de texto se impone, en primer lugar, priorizar los temas previstos en la experiencia curricular o asignatura de un curso determinado, de manera que estos sean ordenados de acuerdo a la programación curricular previa. Sin embargo, esto no resulta una idea cerrada, pues dichos temas pueden ser enriquecidos con otros subtemas que contribuyan con la formación integral de los estudiantes y del lector en general; aunque los mismos no sean contemplados para el ejercicio de la docencia como tal. Las temáticas deben guardar una coherencia entre las necesidades del estudiante beneficiario y el entorno sociocultural que lo circunda.

Posterior a este paso, las temáticas seleccionadas son sometidas a juicio de expertos, o sea, se acude a un equipo de trabajo multidisciplinario que, tomando como referencia la programación curricular del curso, corrobore los contenidos seleccionados y dictamine la pertinencia de los mismos. Una vez recibida la aprobación, es necesario poner manos a la obra. Para ello, el editor o encargado debe trazar determinadas pautas, teniendo en cuenta que la elaboración de un texto universitario es, ante todo, un acto de creación cultural, y como tal debe asumirse.

Cada temática que sea abordada debe contar con un resumen previo, donde se introduzca el tema, se explique de manera sintetizada el objetivo, la estructura y los principales hallazgos del estudio. Para ello, "se hace una selección y condensación de los contenidos claves, donde debe omitirse la información trivial y de importancia secundaria. Un buen resumen debe comunicar las ideas de manera expedita, precisa y ágil" (Universidad Cristóbal Colón, s.f., p. 2).

De la misma manera, deben argumentarse los posibles antecedentes que se correspondan con la temática a desarrollar y, al propio tiempo, explicar cómo se relacionan con la misma. La información procedente de otras fuentes, se cita de 
acuerdo a la norma seleccionada. Una vez desarrollada cada temática, según los criterios acordados, deben agregarse las notas (en caso de existir), las referencias bibliográficas $\mathrm{y}$, por último, la semblanza del autor o autores.

\section{Metodología para seleccionar la información teórica}

Para seleccionar la información teórica adecuada, es imprescindible asumir que, muchas veces, la autenticidad, cantidad y vigencia de la bibliografía seleccionada, condicionan la utilidad teórica, metodológica y práctica del texto.
Actualmente, existe mucha información disponible, ya sea en centros de información u otras vías, como internet. Por tanto, se torna cada vez más fácil acceder a conocimientos nuevos, convenientes y útiles; pero, al mismo tiempo, encontramos mucha información de dudosa calidad y procedencia, por lo que saber escoger los mejores contenidos es un gran reto para los investigadores que conforman el equipo de trabajo.

A continuación, se proponen dos modelos de tabla que facilitan una adecuada gestión bibliográfica.

Tabla 1. Propuesta de tabla para la gestión (obligatoria) de referencias bibliográficas

\begin{tabular}{lcccc}
\hline Fuentes para citas y referencias & $\begin{array}{c}\text { Cantidad } \\
\text { mínima }\end{array}$ & $\%$ & Condición & Vigencia \\
\hline Revistas impresas o en línea con ISSN & 20 & 50 & Obligatoria & \\
Libros impresos o en línea con ISBN & 5 & 15 & Obligatoria & T0 \% últimos \\
Tesis doctorales aprobadas & 2 & 15 & Obligatoria & cinco años \\
Conferencias magistrales & 1 & 10 & Obligatoria & \\
Tesis (maestría, licenciaturas e & 1 & 5 & Obligatoria & \\
ingeniería) aprobadas & 1 & 5 & Obligatoria & \\
Repositorios académicos & 30 & 100 & & \\
Total & & & & \\
\hline
\end{tabular}

Fuente: Elaboración propia

Tabla 2. Propuesta de tabla para la gestión (opcional) de referencias bibliográficas

\begin{tabular}{lcccc}
\hline Fuentes para citas y referencias & $\begin{array}{c}\text { Cantidad } \\
\text { máxima }\end{array}$ & $\%$ & Condición & Vigencia \\
\hline Páginas web & 4 & - & Opcional & \\
Videoconferencias & 4 & - & Opcional & \\
Periódicos impresos o en línea & 2 & - & Opcional & \\
Blogs & 2 & - & Opcional & $100 \%$ \\
Libros y revistas no indizados & 2 & - & Opcional & últimos \\
Investigaciones no publicadas & 1 & - & Opcional & cinco años \\
Entrevistas & 2 & - & Opcional & \\
Datos & 2 & - & Opcional & \\
Otros & 1 & - & Opcional & \\
Total & 20 & - & & \\
\hline
\end{tabular}

Fuente: Elaboración propia 
Estas tablas no representan ideas para los docentes y la sociedad en general. rígidas ni cerradas, más bien flexibles y ajustables a las necesidades curriculares. Representan un punto de partida necesario para la adecuada gestión de referencias bibliográficas. Por ello, la aplicación objetiva de las mismas le atribuiría al libro de texto universitario actualidad, alto nivel de depuración teórica, aval y utilidad, no solo para los estudiantes, sino también

\section{a. Detección de la literatura apropiada}

Para el desarrollo de este paso, se realizará un marco conceptual que básicamente consiste en buscar aquellas teorías, precisiones teóricas o conceptos que sean relevantes para la fundamentación del libro de texto. Para ello, se utilizará el siguiente modelo de ficha bibliográfica (ver Figura 1):

Fecha (fecha en tiempo real): (día/mes/año)

Nombre(s) del(de los) autor(es): Gloria

Apellidos: Fariñas León

Nombre(s) y apellidos del

(traductor; prologuista; ilustrador; coordinador; etc.)

Título del libro/artículo, etc. Psicología, educación y sociedad. Un estudio sobre...

\begin{tabular}{|c|c|c|c|c|c|}
\hline Año: & 2007 & $\begin{array}{l}\text { Lugar de } \\
\text { edición: }\end{array}$ & La Habana, Cuba & $\begin{array}{l}\text { N. }{ }^{\circ} \text { de } \\
\text { edición: }\end{array}$ & 1. ${ }^{\mathrm{a}} \mathrm{ed}$. \\
\hline
\end{tabular}

Editorial: Félix Varela

Página(s) consultada(s): 5

Total de páginas: 224

Código (según sea el caso): Libro versión impresa: ISBN 978-959-07-0586-1

Sitio web (si fuera el caso): (No aplica)

Fecha de acceso (si fuera el caso): (No aplica)

Cita textual: obedece a una casualidad lineal o mecánica, esto habla en parte de la complejidad de esta relación.

Figura 1. Modelo de ficha bibliográfica (resulto). Fuente: Elaboración propia. 
De acuerdo con Sánchez (2008), las fichas bibliográficas constituyen un instrumento imprescindible en el proceso de investigación, particularmente en la fase inicial de consultar los libros o fuentes principales que forman la base del análisis y argumentación del trabajo. La función de una ficha bibliográfica consiste en identificar las fuentes de información que se van a examinar o estudiar para escribir los libros, folletos, artículos de revistas, tesis, etc. (p. 2) Por tanto, facilitan un mejor dominio y manejo de toda la información pertinente para la elaboración del libro de texto universitario.

\section{b. Indagación teórica}

Los autores deben cerciorarse que las teorías a utilizar para el desarrollo de los temas, sean novedosas y atinadas; criticarlas, contrastarlas entre sí y establecer puntos de inflexión y utilidad entre ellas. De acuerdo con Hernández, Fernández y Baptista (2005): “Una teoría es útil porque describe, explica y predice el fenómeno o hecho al que se refiere, además de que organiza el conocimiento al respecto y orienta a la investigación que se lleva a cabo sobre el fenómeno" (p. 60). Y agregan que "los criterios más comunes para evaluar una teoría son: la capacidad de descripción, explicación y predicción; la consistencia lógica; la perspectiva, la fructificación y la parsimonia" (p. 61).

Según Hernández, Fernández y Baptista (2010), los procesos de indagación teórica (revisión de la literatura) son muy importantes gracias a que pueden revelar diferentes grados de desarrollo del conocimiento; en el caso de la elaboración del libro de texto universitario, el equipo de trabajo designado debe corroborar que existe un vacío en el conocimiento por resolver, enfocarse en ello y no desgastarse queriendo "descubrir la rueda".

\section{c. Interpretar las teorías}

En este momento, el profesional podrá valerse de los métodos filosófico, inductivodeductivo, histórico, entre otros. Se trata de realizar un abordaje teórico relevante que ayude a prevenir errores que se hayan cometido en otros estudios, que oriente sobre cómo habrá de llevarse a cabo el estudio, que amplíe el horizonte del estudio y guíe al investigador para que este se centre en su problema evitando desviaciones del planteamiento original. Que conduzca al establecimiento de afirmaciones que más tarde habrán de someterse a prueba en la realidad. Que inspire nuevas líneas y áreas de investigación y que provea de un marco de referencia para interpretar los resultados del estudio (Hernández, Fernández y Baptista, 2010, p. 52).

Al margen de todo ello, interpretar las teorías consultadas es también una actividad propiamente metodológica que debe hacerse con demasiada meticulosidad y creatividad. Para ello, no basta con encontrar la información adecuada, pues lo más importante es que esta información sea bien interpretada y adaptada al contexto sociocultural; si no se puede hacer esto, se impone rechazarla porque no se ajusta a la necesidad teórica y, por tanto, carece de aplicación e implicación sociales.

\section{d. Conclusiones teóricas}

Esta es la parte deductiva, y aunque muchos investigadores prefieren desarrollarla al final de cada temática, también puede hacerse consecutivamente al cierre de cada subtema; sin embargo, se recomienda la primera opción. 
Las conclusiones teóricas son el resultado de la aplicación del método inductivo y, como su nombre lo indica (conclusiones teóricas), se arriba a cierto conocimiento que no es otra cosa que el anclaje o aporte teórico. Este momento es muy importante, porque el profesional debe tener la habilidad de sintetizar ideas y resaltar aquellas que son más convenientes para la obra académica en construcción.

\section{e. Funciones didácticas}

Con la información teórica no basta. Se necesitan libros didácticos que "contribuyan a crear las condiciones para que los estudiantes asimilen los contenidos de la enseñanza... que dicha actividad implica" (Borja, 2005, p. 1).

A propósito, Borja (2005) agregó que al libro de texto le corresponden las siguientes funciones didácticas:

- Informativa. Presentación de la totalidad de la información indicada por el programa de la respectiva asignatura.

- Transformadora. En dos sentidos: 1) reelaboración didáctica de los contenidos; 2) conversión de la actividad puramente cognoscitiva de los estudiantes en actividad transformadora.

- Sistematizadora. Exposición del material docente en una secuencia rigurosa sistematizada, para que el estudiante domine los procedimientos de la sistematización científica.

- De consolidación y de control. Contribución para que los estudiantes se orienten en el conocimiento adquirido y se apoyen en él para realizar la actividad práctica.

- De autopreparación. Formación en los estudiantes del deseo de aprender y de la capacidad de aprender por sí mismos.

- Integradora. Ayuda a los estudiantes para asimilar y seleccionar los conocimientos como un todo único.

- Coordinadora. Aseguramiento del empleo más efectivo y funcional de todos los medios de enseñanza y del uso de medios extradocentes de información masiva.

- Desarrolladora y educadora. Contribución a la formación activa de los rasgos esenciales de la personalidad armónica y desarrollada.

A pesar de que todas estas observaciones se comparten ampliamente y se asumen, resulta necesario complementar con algunas adecuaciones. Como ya se ha dicho, la información contenida en el libro de texto universitario puede salirse de la programación curricular y enriquecerse con otros temas de interés común entre docentes, estudiantes y lectores en general.

Finalmente, las sociedades estudiantiles actuales requieren libros de texto que problematicen, que permitan darle un sentido lógico a la teoría, o sea, que vinculen los aspectos cognitivos, valorativos $\mathrm{y}$ prácticos en un mismo lugar. Textos académicos e innovadores que viabilicen el desarrollo sociocultural del estudiante. "Que transformen la manera de pensar y actuar en la educación" (De Zubiría, 2011, p. 37). Textos originales que conduzcan no solo a identificar, sino a sistematizar y solucionar problemas del contexto sociocultural no resueltos en textos anteriores.

\section{Edición del libro de texto}

Una vez aclarados los aspectos anteriores, y con toda la información investigada, se inician los procedimientos para la edición de la obra. Para ello, es necesario seguir 
un orden metodológico de acuerdo a los siguientes pasos:

\section{a. Estructura del libro de texto}

La estructura del libro de texto estará consignada por los siguientes elementos: portada, contraportada, índice de contenidos, introducción, capítulos (que pueden dividirse en subcapítulos, para evitar que se produzca una carga excesiva de contenidos), notas (si las hubiese), referencias bibliográficas (Pérez, Pupo y Buenaventura, 2011, p. 6) y semblanza de los autores.

Las notas, como su nombre lo indica, son ciertas acotaciones que, por ser de menor trascendencia, deben ubicarse al pie de página o al final del documento. No obstante ello, se recomienda evitarlas siempre que no afecte la claridad e intencionalidad de la información que se aborda. Para la elaboración del libro de texto, se recomienda utilizar las normas APA 6ta. ed., por tanto, en esta modalidad, "se utilizan paréntesis dentro del texto en lugar de notas al pie de página o al final del texto" (Centro de Escritura Javeriano, 2010, p. 4).

Por su parte, la semblanza de los autores es un paso que se puede considerar opcional; sin embargo, se recomienda que, en caso de asumirse, debe aparecer en la página inicial del tema o al final, antes de las referencias. Se sugiere que sea un escrito, de no más de 80 palabras, que informe acerca de nombres $\mathrm{y}$ apellidos del autor, título profesional y grados académicos o científicos. Además, debenaparecer datos como reconocimientos institucionales, principales publicaciones, institución a la que pertenece, etc. Finalmente, correo electrónico, teléfono y alguna otra información que se considere útil y relevante.
Las referencias se ubican con ese mismo nombre (Referencias), al final del documento, de acuerdo a las normas que sean seleccionadas. Se recomienda utilizar las normas APA 6ta. ed. por ser "una de las más conocidas internacionalmente, y tal vez la más utilizada hoy en día" (Centro de Escritura Javeriano, 2010, p. 2). Además, "constituyen el marco de referencia para la presentación de comunicaciones científicas, que se caracteriza por una redacción clara y continua, la ausencia de adjetivos $\mathrm{y}$ afirmaciones que impliquen sesgos de lenguaje, y la escritura en tercera persona o en infinitivo" (Universidad Piloto de Colombia, 2010, p. 1). No obstante, existen otras normas de gran utilidad y difusión mundial, como las ISO, Harvard, Vancouver, entre otras.

\section{b. Tratamiento de imágenes o tablas}

Tanto las imágenes como las tablas deben tener título en el parte superior y la fuente de procedencia en la inferior de la misma. Según De la Vega (2012), las figuras pueden ser:

- Diagramas: pueden describir las relaciones entre partes de un grupo y objeto.

- Dibujos: ayudan a enfatizar cualquier aspecto de una imagen o idea.

- Mapas de puntos: muestran la densidad de una población o alguna otra cosa.

- Mapas sombreados: representan promedio.

- Fotografías: con claridad y calidad profesional.

También pueden ser gráficos de pastel, entre otros; en todos los casos debe especificarse la fuente de procedencia. De ser elaborada por el propio autor, entonces esa información debe especificarse en la parte inferior de la misma. 
Las tablas deben evitarse en lo posible pero, si se deciden utilizar, serán organizadas de acuerdo a un mismo estilo.

\section{c. Etapa de corrección}

La corrección, a diferencia de lo que piensan muchos "avezados", no es un proceso ni automático ni fácil. Es, más bien, una actividad científica, que por ningún concepto debe asumirla el autor. El autor podría ser el peor corrector. Se recomienda hacer una exhaustiva revisión doble. Finalmente, una revisión evaluativa y aprobatoria, donde el corrector de mayor aval emita un dictamen final de conformidad.

Por último, hay que imprimir varias copias (machotes), y en una mesa de trabajo conformada por el editor, correctores, autores y otros especialistas, hacer una revisión minuciosa y crítica de la obra para limar posibles últimos inconvenientes $\mathrm{y}$ autorizar la puesta en valor del libro de texto universitario.

\section{CONCLUSIONES}

Actualmente, la producción de libros de textos universitarios, además de escasa, no es congruente con las necesidades educativas de estos tiempos. Por ello, se destaca la necesidad de producir obras cuyo contenido viabilice mejor, las relaciones académicas entre alumnos, docentes y sociedad.

De la misma manera, las temáticas a abordar en el libro deben ser organizadas a partir de una programación curricular previa, sin que esto signifique una idea cerrada, pues dichas temáticas pueden ser enriquecidas con otros subtemas que contribuyan con la formación integral de los estudiantes, docentes y sociedad.

Para gestionar la información teórica de un libro de texto universitario, se necesita encontrar los contenidos que resulten no solo convenientes, sino relevantes, lo contrario podría traer resultados adversos para todos, especialmente para los alumnos. Por tanto, se hace necesario que la información teórica seleccionada sea novedosa, atinada, criticada, contrastada con otras y demostrar su utilidad en los ámbitos cognitivos, valorativos y prácticos. En tal sentido, esta investigación propone dos tablas que facilitan la gestión de las citas y referencias bibliográficas.

La estructura del libro de texto universitario, según Pérez, Pupo y Buenaventura (2011), debe estar consignada por los siguientes elementos: portada, contraportada, índice de contenidos, introducción, capítulos (que pueden dividirse en subcapítulos, para evitar que se produzca una carga excesiva de contenidos), notas (si las hubiese), referencias y semblanza de los autores.

La aplicación de esta propuesta metodológica, garantiza que los libros de textos universitarios sean editados no solo con la calidad académica requerida, sino de forma controlada. Se garantiza que estudiantes, docentes y sociedad accedan a textos despojados de errores conceptuales evitables, estereotipos ideológicos, obras abiertas fundamentadas en la construcción crítica de los conocimientos.

Del mismo modo, esta propuesta facilita que los libros de texto gocen de mucha integralidad, pues además de facilitar la interacción interdisciplinar y la interconexión del saber científico, el conocimiento compartido y la mirada globalizadora, sirve para construir (y reconstruir) la cultura, aunque las mismas no sean objeto de estudio de la academia en sí.

Finalmente, esta metodología garantiza que la creación del libro de texto universitario se lleve a cabo con un margen de error mínimo, ya que su edición depende exclusivamente de la objetivación cultural del currículo en todas 
sus dimensiones, de la construcción de nuevas concepciones y prácticas sobre su uso en la educación,asícomo dela concreciónderelaciones entre discursos y representaciones sociales (Escolano, 2000, citado por Celis, 2001, p. 3).

\section{REFERENCIAS}

Acero, F. (2008). Estructura del libro de texto universitario: un análisis de textos de álgebra lineal. (Tesis de maestría). Recuperado de http://www.udesa.edu. ar/files/MaeEducacion/Microsoft $\% 20$ Word\%20-\%20Resumen\%20Acero.pdf

Borja, I. (2005). Caracterización del libro de texto de castellano para la educación primaria colombiana: tipología $\mathrm{y}$ componentes. Revista Iberoamericana de Educación, 2(36), 1-14. Recuperado de http://www.rieoei.org/ deloslectores/937Alarcon.PDF

Celis, Z. (2011). Los libros de texto gratuitos en México. Vigencia y perspectivas. Recuperado de http://www.comie.org. $\mathrm{mx} /$ congreso/memoriaelectronica/ v11/docs/area_13/2420.pdf

De la Vega, M. (2012). Introducción al Estilo APA, 6ta. ed. Tablas y Figuras. Recuperado de http://investigacion.casagrande.edu. ec/wp-content/uploads/2013/10/3.TABLAS-y-FIGURAS-APA-6ta.-ed..pdf

De Zubiría, J. (2011). Las competencias argumentativas. La visión desde la educación. Bogotá: Cooperativa Editorial Magisterio.

Duarte, L. y Torres, G. (2010). Guía para la presentación de reporte final de trabajos de grado en la Universidad Piloto de Colombia, basado en las normas APA. Recuperado de http://www.unipiloto. edu.co / d e s carga s / N o r mas $\% 20$ APA\%20Sexta\%20Version.pdf

Edmondson, A. \& Nembhard, I. (2010) La innovación y el aprendizaje en los equipos: retos convertidos en beneficios. Harvard Deusto, (195), 64-72. Recuperado de http://www.harvarddeusto.com/articulo/La-innovacion-yel-aprendizaje-en-los-equipos-retosconvertidos-en-beneficios

Fernández, A. (2005). La importancia de ser llamado "libro de texto". Hegemonía y control del currículo en el aula. Argentina: Universidad Nacional de la Patagonia Austral. Recuperado de http://www.biblioteca.unlpam.edu.ar/ pubpdf/ieles/n04a10ferrero.pdf

Hernández, R., Fernández, C. y Baptista, P. (2005). Metodología de la investigación. Editorial Pablo de la Torriente: La Habana, Cuba.

Hernández, R., Fernández, C. y Baptista, P. (2010). Metodología de la investigación. (Quinta ed.) México D.F: McGraw-Hill.

Martínez, J. (2006). ¿De qué hablamos cuando hablamos de los libros de texto? Valencia, España: Universitat de València. Recuperado de http://www.uv.es/ bonafe/documents/libros\%20texto.pdf

Martínez, J. (2008). Los libros de texto como práctica discursiva. Revista de la Asociación de Sociología de la Educación, 1(1), 62-73. Recuperado de http://dialnet.unirioja.es/servlet/ articulo?codigo $=2793153$

Pérez, M., Pupo, S. y Buenaventura A. (2011). Metodología para la elaboración de libros de texto en la enseñanza del inglés con fines turísticos. Ciencias Holguín, 17(2), 1-10. Recuperadodehttp://www.redalyc. org/articulo.oa?id=181522301011

Sánchez, R. (2008). Guías para elaborar fichas bibliográficas en la redacción de ensayos, monografías y tesis. Puerto Rico: Universidad de Puerto Rico. Recuperado de http://www. 
blancopeck.net/GU\%C3\%8DAS.pdf Student PIRGs. (2012). Open Textbooks: More Information. PIRGs, USA. Recuperado de http://www.studentpirgs.org/opentextbooks/about

Universidad Cristóbal Colón. (s.f.). Documentos universitarios. La divulgación científica y tecnológica. Recuperado de https:// www.kennedy.edu.ar/DocsEsc81/ Material\%20Bibliogr\%C3\%A1fico/ Informe, $\% 20$ art $\%$ C $3 \%$ ADculo, $\% 20$ ensayo.pdf

( ) Los autores. Este artículo es publicado por la Revista Digital de Investigación en Docencia Universitaria del Área de Investigación de la Dirección de Calidad Educativa, Universidad Peruana de Ciencias Aplicadas. Este es un artículo de acceso abierto, distribuido bajo los términos dela Licencia Creative Commons Atribución-CompartirIgual 4.0 Internacional.(http://creativecommons.org/licenses/by-sa/4.0/), que permite el uso no comercial, distribución y reproducción en cualquier medio, siempre que la obra original sea debidamente citada. 